\title{
Asset Management based on Internet of Things
}

\author{
Sanam Kadge \\ Assistant Professor, \\ Department of \\ Information \\ Technology, M.H. \\ Saboo Siddik College \\ of Engineering, \\ Mumbai, India
}

\author{
Chaudhary Hasan \\ Ahmed \\ Student (B.E), \\ Department of \\ Information \\ Technology, M.H. \\ Saboo Siddik College \\ of Engineering, \\ Mumbai, India
}

\author{
Abdul Qadir Zilani \\ Student (B.E), \\ Department of \\ Information \\ Technology, M.H. \\ Saboo Siddik College \\ of Engineering, \\ Mumbai, India
}

\author{
Yash Jain \\ Student (B.E), \\ Department of \\ Information \\ Technology, M.H. \\ Saboo Siddik College \\ of Engineering, \\ Mumbai, India
}

\begin{abstract}
Though IT industry is rapidly growing with the use of recent technologies, IT assets management is semi-automated and unmanaged. Lots of research is carried on and existing asset management software have become intelligent by integrating it with $\mathrm{AI}$ (artificial intelligence) but as manual operation is required to manage the system it leads to poor efficiency and security for small and medium enterprise. It can be solved using self-management. IoT provides solution by enabling the design and development of Systems that can adapt themselves to meet the requirements without manual intervention. Internet of Things is the scenario where billions of devices and objects are being embedded with sensors and chips. As these devices gain the ability to communicate using the IoT, the resulting information networks deliver an exponential increase in insight about customer behavior. Organizations can use this insight to create new operating and business models, improve business processes and reduce costs, strengthen security and manage risk, and manage the change that comes with a dynamic environment. When you capitalize upon the insight-rich data that is generated by the Internet of Things, you can address customer service in a manner that has not been possible before and hence is transformative.
\end{abstract}

\section{Keywords}

Asset management, Internet of Things (IoT), Cloud computing.

\section{INTRODUCTION}

The lifecycle of an asset by the traditional method is to submit a new request for asset, get manager approvals, procurement submission where they get price negotiations from a pre-defined vendor list and then the PO is sent to the vendor. This type of system is very time consuming. All the hardware, software and their related PO e.g. invoices, licenses are scattered everywhere which is difficult to manage. This system is more prone to bugs or errors and sometimes the approach to various problems is unstructured. IT asset involves gathering of detailed software and hardware inventory information which can be further used to make decisions about their purchases and redistribution. Managing asset helps organizations to manage their assets more effectively in addition it also saves time and money by avoiding asset purchases which is not necessary. Asset management in IT field is the set of business practices related to financial and inventory functions to support decision making and also help in life cycle management for the IT environment. Organizations that follow, maintain and develop an effective IT asset management minimize the incremental risks and related costs of advancing IT portfolio infrastructure projects based on old and redundant information. The data-rich, two-way communication that makes up the Internet of Things is changing rapidly. The Internet of Things originally linked computers to computers. Now billions of devices are connected to the web: smart phones, automobiles, light bulbs, utility meters and remote sensors. In the coming decade, the quantity and types of devices that are linked to the web-and the apps that will run on this expanding Internet of Things-will explode with growth, driving a new Internet revolution[1] . It is estimated that the IoT has the potential to create an economic impact of $\$ 2.7$ trillion- $\$ 6.2$ trillion (USD) annually by the year 2025[2] .As you take action to manage this sudden rise in disruptive technologies, your teams will be challenged to react quickly - especially if your organization is large or complex. The organizations connect devices, data, applications, central systems and people to create new business opportunities and solve business pains. For them, the Internet of Things is not about devices; it is about imagination. How can you better serve a remote group of clients? How can you anticipate people's needs before they are articulated? How can you unlock static data and make it work for your customers? Technologically, all Internet of Things projects will involve devices, messaging from those devices, the data those messages carry, and the applications built upon that interchange. Yet the way those components combined are both simple and brilliant. It is a great potential for even small and medium industries in the growth of the business using asset management with IoT.

\section{IDENTIFYING THE VARIOUS PROBLEMS IN ASSET MANAGEMENT}

Fortunately, the problems necessitating the implementation of a comprehensive IT asset management strategy can be identified easily. Unfortunately, however, far too many Engineering organizations cannot answer the following questions with a certain acceptance degree:

- How many computer or laptop the organization needs?

- Current location of the asset?

- Did you purchased the annual maintenance and support for the unused and used assets?

- Are you purchasing currently non-usable upgrades for IT assets?

- What software applications run on each PC?

- Are any of these pirated or illegal copies? 
- $\quad$ Can IT assets currently not being used, be redeployed or put in the scrap? if needed, who need them?

- Can you reclaim unused software license?

- What is the configuration of the asset? (primary and secondary memory, processor speed etc.)?

- Which departments are responsible for a particular asset?

Organizations cannot answer any or all of the above questions due to problems in both the IT department, as well as in other departments responsible for reporting accounting and financial data regarding the IT assets. Typically, the multi-departmental problems from not having updated information. These most probably will include:

- Problems related to re-usage.

- Not able to develop an appropriate migration strategy.

- Problems related to forecasting and budgeting.

- $\quad$ Not able to pass proper audits

- Problems in assets linked to certain contracts and agreements.

- Moving, adding and editing problems.

- $\quad$ Not able to provide accurate management reports.

\section{RISKS TO EFFECTIVE ASSET MANAGEMENT}

Security risks are the events which are often, represented as any event that compromises the assets, objectives and operations of an organization. Some security risks that are directly related to it are

- Improper risk management

- $\quad$ Lack of control over services

- Improper maintenance

- Improper manual operation

- $\quad$ Lack of Intelligence to the system

\section{INTERNET OF THINGS (IoT)}

IoT helps in managing large data collection for asset management through value chain. For example, the asset itself will stream that data to a central system where engineers can see it and put in order before the asset fails completely [3]. Also it will help for big data analysis and performing operations that were not possible to find without IoT.

Listed below are the reasons why Iot is beneficial for the industries and manufacturers

- Requires less human interaction and dependency. IoT controls the data workflow and can work with less human interactions. It will require less humans and cost to manage the workflows as most of the system will be automated
- Better maintenance schedule. IoT can be used to maximize uptime reliability to ensure that orders can be fulfilled quickly. Maintenance makes it easy to schedule downtime, and maintenance can be scheduled around by proper planning thereby avoiding missed deadlines.

- Easy tracking of assets. A program starts with a central database that keeps track of work orders, asset conditions and other data needed to make decisions regarding the management of assets. It's very much necessary for the business to know from where the asset is coming in order to cut the cost. Asset management along with IoT can help you to find out the number of resources required for the activities so that you can divert the resource to it and handle further investments to executives.

\begin{tabular}{|c|c|}
\hline DEVICE MANAGEMENT & $\begin{array}{c}\text { RESPONSIVE,SCALABLE } \\
\text { CONNECTIVITY }\end{array}$ \\
\hline $\begin{array}{l}\text { Using this service, you can perform } \\
\text { actions on device like receive } \\
\text { device metadata and diagnostics, } \\
\text { perform bulk device removal or } \\
\text { addition and rebooting or updating } \\
\text { firmware }\end{array}$ & $\begin{array}{l}\text { Industry standard MQTT protocol } \\
\text { is used for connection between } \\
\text { applications and devices. MQTT is } \\
\text { designed for real time exchange of } \\
\text { data to and from the devices }\end{array}$ \\
\hline SECURE COMMUNICATION & $\begin{array}{l}\text { STORAGE AND ACCESS TO } \\
\text { DATA }\end{array}$ \\
\hline $\begin{array}{l}\text { Securely receive data from and } \\
\text { send commands to devices. We use } \\
\text { MQTT with TLS to secure } \\
\text { communication our service and } \\
\text { your device }\end{array}$ & $\begin{array}{l}\text { You can opt to store data for any } \\
\text { period of time. You can have } \\
\text { access to historical and real time } \\
\text { data for your device }\end{array}$ \\
\hline
\end{tabular}

Figure 1. Benefits of IoT in Asset Management

\section{PROPOSED SYSTEM}

The Internet of Things is already creating positive workflow for business and industries in many fields like healthcare, government, insurance, utilities, banking and in the retail and wholesale sectors[4].

Exploring the opportunities that come with Internet of Things for customers and businesses. Discover four ways organizations are growing their business by creating innovative services for clients:

- Better control over existing services and resources

- $\quad$ Extending new services or services to new markets and new places.

- Services optimization for clients

- Monetizing services clients care about and thus gaining profits. 
MOBILE PROCESSES

\section{BACKEND SYSTEM}

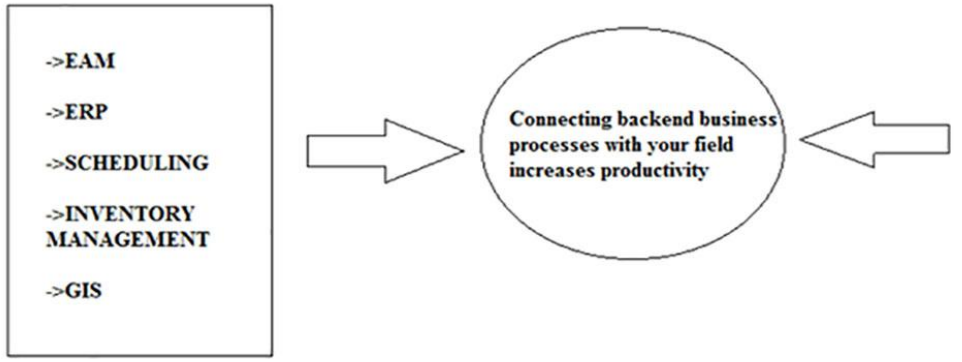

$\rightarrow$ WORK ORDER MANAGEMENT

$\rightarrow$ ROUNDS AND CONDITION MONITORING

$\rightarrow$ INVENTORY

MANAGEMENT

$\rightarrow$ ASSET AUDITING

$\rightarrow$ CALIBRATIONS

$\rightarrow$ SHUTDOWN AND TURNAREOUNDS

Figure 2. Connectivity between backend and mobile processes

\subsection{Organizations}

Organizations ensure that the data is accessible only from your apps. Once registered, devices and API keys are bound to the organization. When an application connects to the service, it registers with the organization that has the API key.

For security it is impossible for other organization communication within the Internet of Things eco-system, intentional or otherwise[5]. To transmit data between two organizations, it needs to explicitly create two applications, one within organization, to communicate with each other to act as a relay between the organizations.

\subsection{Applications}

- An application is anything that has a connection to the internet and wants to interact with data from devices and/or control the behavior of those devices in some manner.
- Applications identify themselves to the IoT with an API key and a unique application ID.

\subsection{Devices}

- A device can be anything which has an internet connection and has data it wants to get into or from the cloud.

- A device is not able to directly interact with other devices due to security reasons.

- Devices are able to accept data and commands from applications.

- Devices uniquely identify themselves to the IoT with an authentication API that will only be accepted for that device.

- Devices must be registered before they can connect to the IoT.

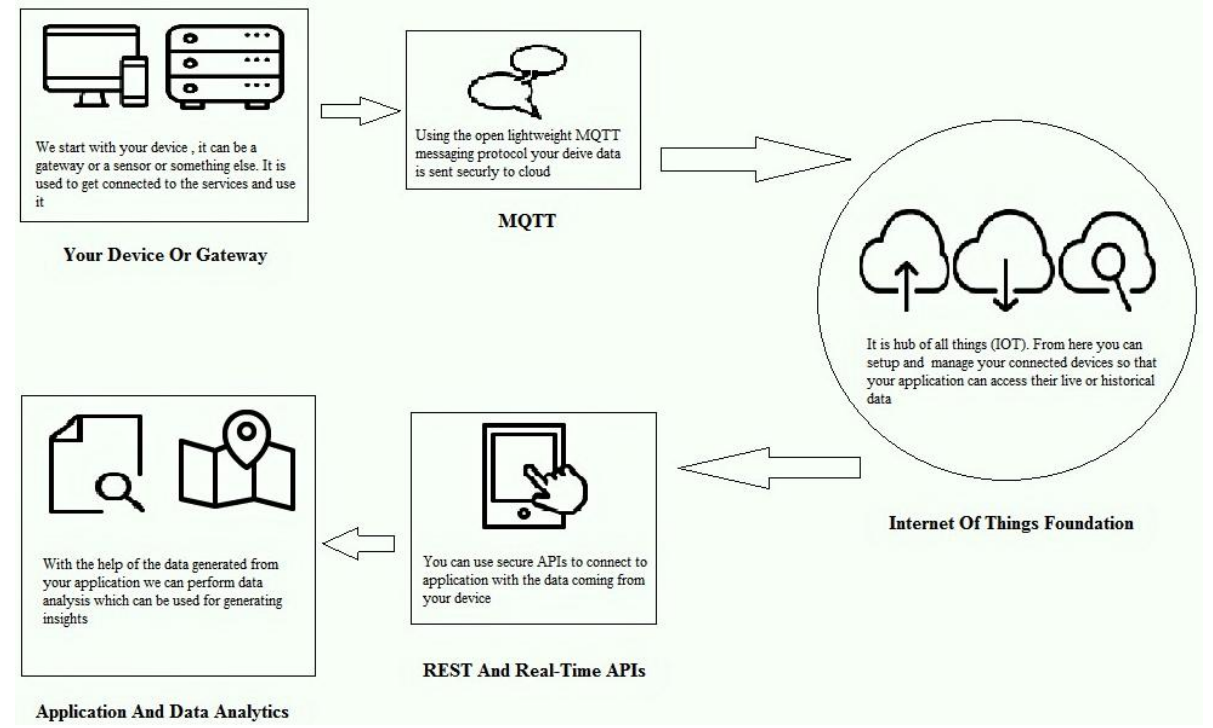

Figure 3. Workflow of IoT 


\section{IMPLEMENTATION}

One need to stay competitive by getting the most out of their assets with asset operation and maintenance. With the right system and processes in place, the organization can help ensure reliability and safety, migrate risks and reduce maintenance costs [6]. As a result, the asset operations and maintenance program can establish a series of steps that help ensure an asset retains to a state in which it can perform its function. Many organizations try to reduce IT costs while increasing the flexibility and dependency of their core business software and systems. Cloud is one of the best solutions which integrate seamlessly with the solutions and eliminate the need for major IT investments; this makes them ideally suited for small and medium businesses and large corporations willing to use the Internet of Things (IoT) and optimize asset management. These are needed more than ever as IoT adoption benefits are huge thanks to reductions in the size and prices of sensors, better adoption of Big Data technology along with systematic analytics, and the assignment of IP addresses for devices. It has been estimated that there will be around 50 billion to 75 billion connected devices with the help of internet (IoT) by 2020[6]*.This rapid adoption of mobile devices - combined with the IoT - is transforming how asset management is conducted. In many industries, technicians no longer roam entire sites with clipboards, manually writing down readings from machines and serves for requesting maintenance. Instead, the IoT is connecting people, machines, and things and enabling a seamless flow of information for real-time decision making. This continuous generation of data and data driven intelligence from the IoT ecosystem is helping companies in some or the other way to enhance their business processes and operations maintenance. Software can help asset management analyze vast amounts of asset related data flowing from various sources and combine it with the latest back-end transactional data. With the help of the predictive capabilities of the software, companies can easily increase their operational efficiency by addressing pending equipment failures. This will finally allow them to reduce or eliminate asset downtime.

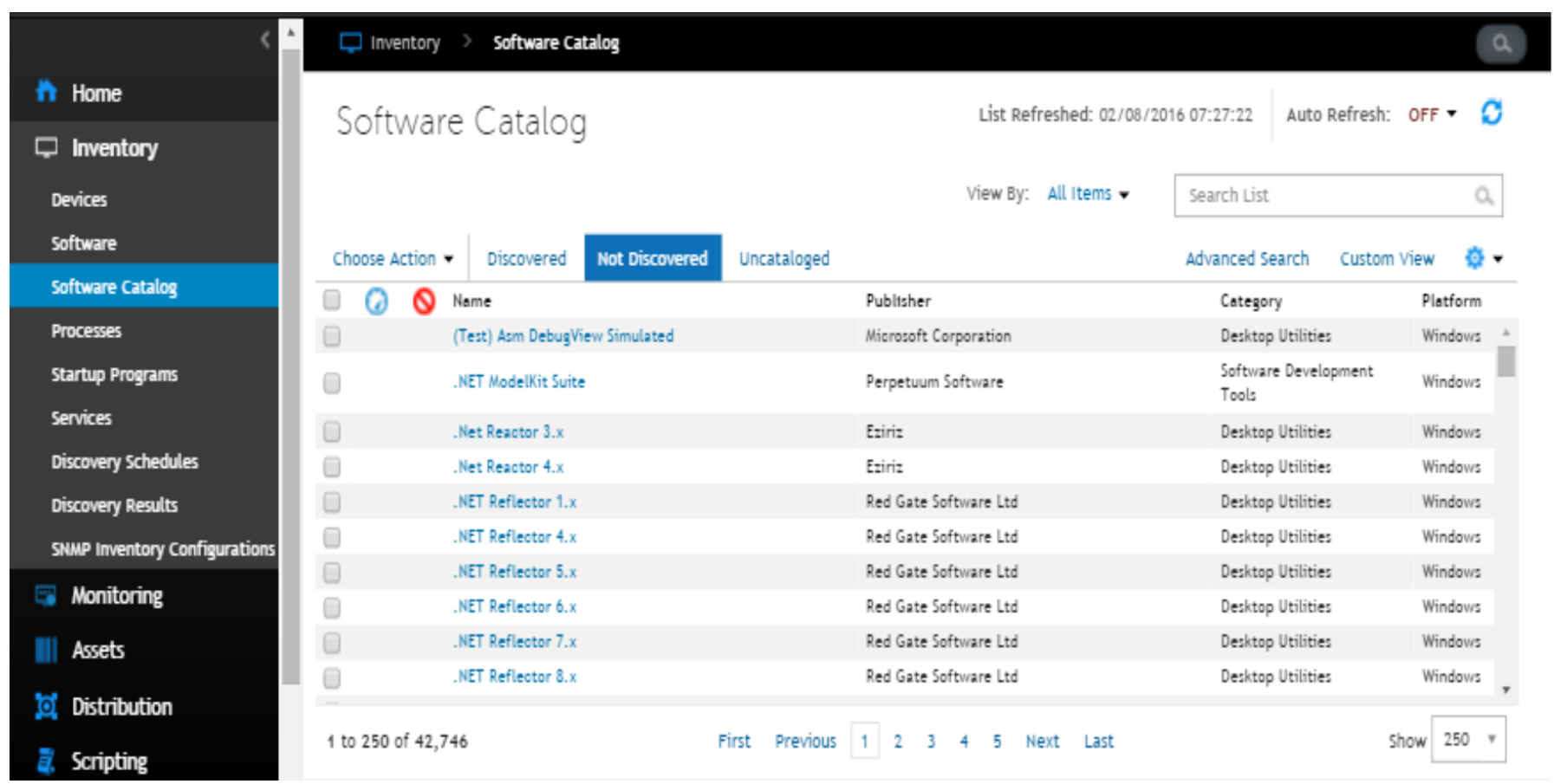

Figure 4. Implementation Of Management Site In Desktop

In the above figure, the softwares with different publishers and category can be seen in the desktop version of the asset management inventory system.Adminstrator can perform various actions and search from the list. 

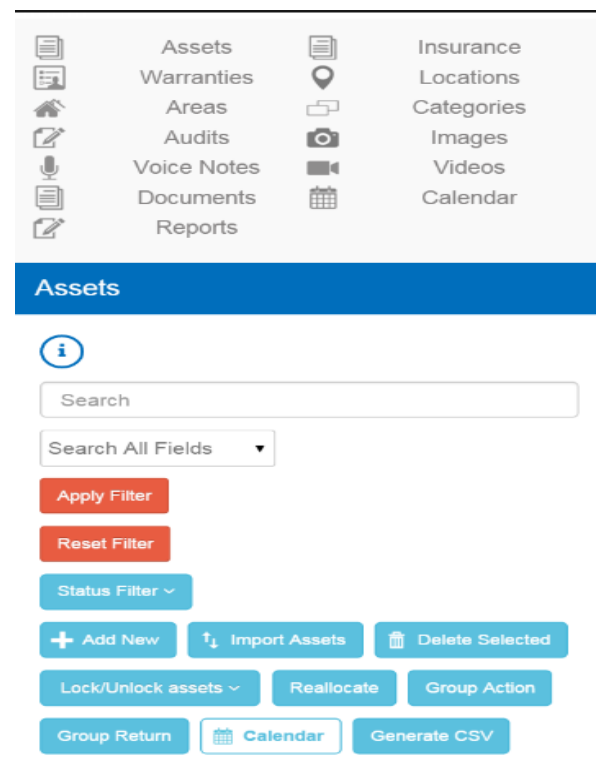

Figure 5. Implementation Of Management Site In Mobile

In the above figure,asset management operation by mobile devices is displayed to the users.Adminstrator can search the assets and also apply filters to perform various actions for the asset management

\section{RESULT ANALYSIS}

The following result analysis were obtained on various points regarding asset management based on the comparison before and after the implementation of IoT based on figure 6

- Manpower is reduced by $75 \%$.

- Downtime and production is reduced by 20 to $30 \%$.
- Preventable Failures is reduced by $90 \%$.

- Maintenance Backlog Time is reduced by $60 \%$.

- Inventory Carry Cost is reduced by 5 to $10 \%$.

- Work Capacity is increased by 10 to $20 \%$.

- Labor Productivity is increased by $50 \%$.

- Idle Time And Overtime is reduced by 42 to $58 \%$.

- $\quad$ Rework is reduced by 15 to 20

Table 1 . Analysis of different points before and after IoT

\begin{tabular}{|c|c|c|c|}
\hline Sr.no & Analysis Points & Before IoT & After IoT \\
\hline 1 & Manpower & $436 \mathrm{unit} \mathrm{hrs} / \mathrm{month}$ & 109 unit hrs/month \\
\hline 2 & Downtime & $20 \mathrm{hrs} / \mathrm{month}$ & $2 \mathrm{hrs} / \mathrm{month}$ \\
\hline 3 & Preventable Failures & $18 \mathrm{hrs} / \mathrm{month}$ \\
\hline 4 & Maintenance Backlog Time & $50 \mathrm{hrs} / \mathrm{month}$ & $20 \mathrm{hrs} / \mathrm{month}$ \\
\hline 5 & Inventory Carry Cost & INR $5,00,000 \mathrm{per}$ month & INR $4,60,000$ per month \\
\hline 6 & Work Capacity & $50 \mathrm{hrs} /$ week & $58 \mathrm{hrs} /$ week \\
\hline 7 & Labor Productivity & 50,624 units & 75,302 units \\
\hline 8 & Idle Time And Overtime & $12 \mathrm{hrs} /$ week & $7 \mathrm{hrs} /$ week \\
\hline 9 & Rework & $35 \mathrm{hrs} /$ week & $28 \mathrm{hrs} /$ week \\
\hline
\end{tabular}

\section{CONCLUSION}

For a company dealing with asset management, staying competitive and providing amazing customer service is of at most importance. This requires access to correct and real-time asset lifecycle information at all time, regardless of asset ownership or location. On-premise and cloud applications working together - and combined with asset-specific content by a cloud platform - form the basis of any asset management with IoT.

The future of asset management depends on a network-based asset management solution driven by IoT platforms that can brings together the manufacturer, services and logistics providers along with the operators of the world. This chain will be the key to maximizing asset value, reducing time and errors, and improving customer service. 


\section{REFERENCES}

[1] H. Li, X. Liu, W. Sun, "Analytics-Driven Asset Management, IBM Journal of Research and Development, January/March 2011.

[2] R. Davies, J. Dieter \& T. McGrail, "The IEEE and Asset management: A Discussion Paper", IEEE PES Conference, Detroit, July 2011

[3] Nasui, D. Sgarciu.V.Cernian, A., "Cloud-based application development platform for secure, intelligent, interlinked and interactive infrastructure," Applied Computational Intelligence and Informatics (SACI), 2013

[4] IEEE Lin, S., GAO, J., Koronios, A., and Chanana, V., "Developing a data quality framework for asset management in engineering organizations," International Journal of Information Quality, 1 (1), 2007, p.100-126.

[5] B. Zhao, W. Hao, W.S. Hao, X.M. Dong, "Research and development of equipment management system based on cloud computing", Manufacturing Automation, vol. 33, pp. 48-51, January 2011

[6] ISO 55000 (Draft): Asset Management - Overview, principles and terminology.

[7] John E. Provo, IT Asset Management - an Integral Part of an Overall Asset Management Strategy.

[8] http://go.sap.com/solution/lob/asset-management.html
[9] http://www.ibm.com/cloudcomputing/us/en/interconnect/agenda/internetofthings

[10] https://internetofthings.ibmcloud.com/\#/. http://www.genesissolutions.com/asset-management-tobe-a-key-in-internet-of-things-manufacturingdeployments/.

[11] http://www.genesissolutions.com/asset-management-tobe-a-key-in-internet-of-things-manufacturingdeployments/.

[12] https://software.dell.com/techbrief/dell-kace-remotemanagement-with-intel-vpro-technology874055/

[13] John E. Provo, "IT Asset Management — an integral part of overall asset management strategy" IEEE Conference 2012.

[14] Rhys Davies, "The IEEE and Asset Management: a Discussion Paper" Smart Asset Management Solutions, 23 Pendinas, Wrexham. LL11 3BQ

[15] Tony McGrail, "The IEEE and Asset Management: a Discussion Paper" National Grid. Electricity Distribution and Generation, 40 Sylvan Road. Waltham, MA. 02451 USA

[16] The Asset management Conference, IAM \& IET London,November 2010 . http://conferencestheiet.org/asset 